\title{
Neutrino Signals from Annihilating/Decaying Dark Matter in the Light of Recent Measurements of Cosmic Ray Electron/Positron Fluxes
}

\author{
Junji Hisano, ${ }^{1,2}$ Masahiro Kawasaki,,${ }^{1,2}$ Kazunori Kohri, ${ }^{3}$ and Kazunori Nakayama ${ }^{1}$ \\ ${ }^{1}$ Institute for Cosmic Ray Research, University of Tokyo, Kashiwa 277-8582, Japan \\ ${ }^{2}$ Institute for the Physics and Mathematics of the Universe, University of Tokyo, Kashiwa 277-8568, Japan \\ ${ }^{3}$ Physics Department, Lancaster University, Lancaster LA1 4YB, UK
}

(Dated: October 24, 2018)

\begin{abstract}
The excess of cosmic-ray electron and positron fluxes measured by the PAMELA satellite and ATIC balloon experiments may be interpreted as the signals of the dark matter annihilation or decay into leptons. In this letter we show that the dark matter annihilation/decay which reproduces the electron/positron excess may yield a significant amount of high-energy neutrinos from the Galactic center. In the case, future kilometer-square size experiments may confirm such a scenario, or even the Super-Kamiokande results already put constraints on some dark matter models.
\end{abstract}

Recently the PAMELA satellite experiment reported a clear excess of the positron flux from the expected background [1], and the ATIC [2] and PPB-BETS [3] balloon experiments have shown the rise in the total electron and positron flux. The HESS collaboration also released a data of the electron flux [4], which is consistent with ATIC/PPB-BETS results. While those results may be explained by the contribution from the pulsar(s) [5, 6], they may be interpreted as the signatures of the annihilation/decay of the dark matter. Many papers have appeared on the later subject [7, 8].

If the observed electron/positron excesses come from the dark matter annihilation, a large annihilation cross section of order of $\sim 10^{-24}-10^{-23} \mathrm{~cm}^{3} \mathrm{~s}^{-1}$ is required, slightly depending on the annihilation mode. Otherwise, a huge boost factor must be introduced, especially when we stick to the annihilation cross section of $\sim 3 \times 10^{-26} \mathrm{~cm}^{3} \mathrm{~s}^{-1}$, which accounts for the present dark matter abundance under the standard thermal relic scenario [9]. The annihilation/decay of the dark matter also yields gamma rays, anti-protons and synchrotron radiations in the Galaxy. Some models proposed to explain the electron/positron excesses may be disfavored due to the saturation of those observed limits. It was also shown that the dark matter annihilation scenario as an explanation of the positron excess is constrained by the big-bang nucleosynthesis [10]. Thus, it is important to look for observational signatures of the annihilation/decay of the dark matter which may be related to the electron/positron fluxes in order to distinguish or exclude some particular scenarios.

In this letter, we evaluate the neutrino flux from the Galactic center, which come from the dark matter annihilation/decay into leptons. Such dark matters are favorable since excess is not observed in the anti-proton cosmic rays spectrum. we point out that observations of high-energy neutrinos arising from the dark matter annihilation/decay into leptons at the Galactic center can provide constraints even at the present stage, and future neutrino telescope projects in the northern hemisphere, such as KM3NeT, will be useful for a cross check of the dark matter scenario, if the currently observed elec- tron/positron excesses truly originate from the dark matter. In particular, we show that the study of neutrinoinduced up-going muon flux is very important for a heavy dark matter with mass of order of a few $\mathrm{TeV}$ indicated by ATIC/PPB-BETS results, as opposed to the case of rather light dark matter, which was studied in Ref. 11] for the specific model of decaying gravitino dark matter with $R$-parity violation.

First, we briefly review the electron and positron flux produced by the dark matter annihilation/decay [12]. Since high-energy electrons and positrons produced by the dark matter annihilation/decay lose their energy quickly due to the synchrotron emission induced by the Galactic magnetic field and inverse Compton processes with CMB photons and star light, they can reach to the Earth only from the region within a few kpc. The propagation of electrons and positrons is described by the following diffusion equation,

$$
\begin{aligned}
\frac{\partial}{\partial t} f(E, \vec{x})= & K(E) \nabla^{2} f(E, \vec{x}) \\
& +\frac{\partial}{\partial E}[b(E) f(E, \vec{x})]+Q(E, \vec{x}),
\end{aligned}
$$

where $f(E, \vec{x})$ denotes the electron and positron number density at $\vec{x}$ with energy $E$. The flux at the Earth $(\vec{x}=$ $\left.\vec{x}_{\odot}\right)$ is given by $\Phi_{e^{-}, e^{+}}^{(\mathrm{DM})}\left(E, \vec{x}_{\odot}\right)=(c / 4 \pi) f\left(E, \vec{x}_{\odot}\right)$ with the speed of light $c . K(E)$ and $b(E)$ represent the diffusion constant and energy-loss rate, respectively. The source term $Q(E, \vec{x})$ is given by

$$
Q(E, \vec{x})=\frac{1}{2} \frac{\rho^{2}(\vec{x})}{m_{\chi}^{2}} \sum_{F}\langle\sigma v\rangle_{F} \frac{d N_{F}^{\left(e^{-}, e^{+}\right)}}{d E},
$$

for the case of annihilation, and

$$
Q(E, \vec{x})=\frac{\rho(\vec{x})}{m_{\chi}} \sum_{F} \Gamma_{F} \frac{d N_{F}^{\left(e^{-}, e^{+}\right)}}{d E},
$$

for the case of decay. Here, $\rho(\vec{x})$ is the mass density of the dark matter, $m_{\chi}$ is the dark matter particle mass, $\langle\sigma v\rangle_{F}$ and $\Gamma_{F}$ are the annihilation cross section and 
the decay rate into the final state $F$ respectively, and $d N_{F}^{\left(e^{-}, e^{+}\right)} / d E$ is the fragmentation function of the final state $F$ into electrons/positrons. The steady-state solution of this equation can be obtained semi-analytically with a cylinder-like boundary condition 13].

Fig. 1 shows positron fraction (top) and total electron and positron flux from the dark matter annihilation (bottom). We take the mass and annihilation cross section as $m=0.7 \mathrm{TeV}$ and $\langle\sigma v\rangle=5 \times 10^{-24} \mathrm{~cm}^{3} \mathrm{~s}^{-1}$ for the mode into $e^{+} e^{-}$(solid), $m=1 \mathrm{TeV}$ and $\langle\sigma v\rangle=$ $1.5 \times 10^{-23} \mathrm{~cm}^{3} \mathrm{~s}^{-1}$ for the mode into $\mu^{+} \mu^{-}$(dashed), $m=1.2 \mathrm{TeV}$ and $\langle\sigma v\rangle=2 \times 10^{-23} \mathrm{~cm}^{3} \mathrm{~s}^{-1}$ for the mode into $\tau^{+} \tau^{-}$(dotted). We have added a background flux given in Ref. [12] with normalization fixed by hand. Also plotted are results of PAMELA [1], ATIC [2], BETS [14] and PPB-BETS [3]. We can see that these models well fit the observed data. Typical cross section into leptons which reproduces the observational data is around $\langle\sigma v\rangle \sim 10^{-23} \mathrm{~cm}^{3} \mathrm{~s}^{-1}$, which may be the result of Sommerfeld enhancement [15]. Quite similar results are obtained for the case of decaying dark matter. In that case, the typical decay rate into leptons should be $\sim 10^{-26} \mathrm{~s}^{-1}$ with the mass around 1.5-3.0 TeV.

Now let us discuss the production of neutrinos from the annihilation/decay of the dark matter particle. As is already mentioned, dark matter annihilation/decay directly into leptons are more favored, and the typical annihilation cross section and decay rate required for explaining the electron/positron excesses are $\langle\sigma v\rangle \sim$ $10^{-23} \mathrm{~cm}^{3} \mathrm{~s}^{-1}$ for the case of annihilation, and $\Gamma \sim$ $10^{-26} \mathrm{~s}^{-1}$ for the case of decay. When the dark matter is $S U(2) \times U(1)$ singlet and annihilates/decays into left-handed charged leptons, the dark matter also annihilates/decays into neutrinos with same cross section/decay rate. Furthermore, charged leptons $(\mu, \tau)$ produced in the annihilation/decay of the dark matter decay into neutrinos. Thus it is natural to expect that when the dark matter mainly annihilates or decays into leptons, it also produces comparable amount of neutrinos. Interestingly, such a value of the cross section is close to the upper bound obtained from the neutrino flux assuming that the dark matter totally annihilates into neutrinos [16].

The possible production processes of neutrinos are direct production $\left(\chi(+\chi) \rightarrow \nu_{i}+\bar{\nu}_{i}\right)$ where $i=1,2,3$ distinguishes flavors and the decay of primary $\mu$ 's and $\tau^{\prime}$ 's $\left(\mu^{-} \rightarrow \nu_{\mu}+\bar{\nu}_{e}+e^{-}\right.$, etc. $)$which are directly produced by the dark matter annihilation/decay $(\chi(+\chi) \rightarrow$ $\left.\mu^{-}+\mu^{+}, \tau^{-}+\tau^{+}\right)$. The neutrino flux at the Earth coming from the Galactic center is evaluated by

$$
\frac{d F_{\nu_{i}}}{d E}=\frac{R_{\odot} \rho_{\odot}^{2}}{8 \pi m_{\chi}^{2}}\left(\sum_{F}\langle\sigma v\rangle_{F} \frac{d N_{F}^{\left(\nu_{i}\right)}}{d E}\right)\left\langle J_{2}\right\rangle_{\Omega} \Delta \Omega,
$$

for the case of annihilation, and

$$
\frac{d F_{\nu_{i}}}{d E}=\frac{R_{\odot} \rho_{\odot}}{4 \pi m_{\chi}}\left(\sum_{F} \Gamma_{F} \frac{d N_{F}^{\left(\nu_{i}\right)}}{d E}\right)\left\langle J_{1}\right\rangle_{\Omega} \Delta \Omega,
$$
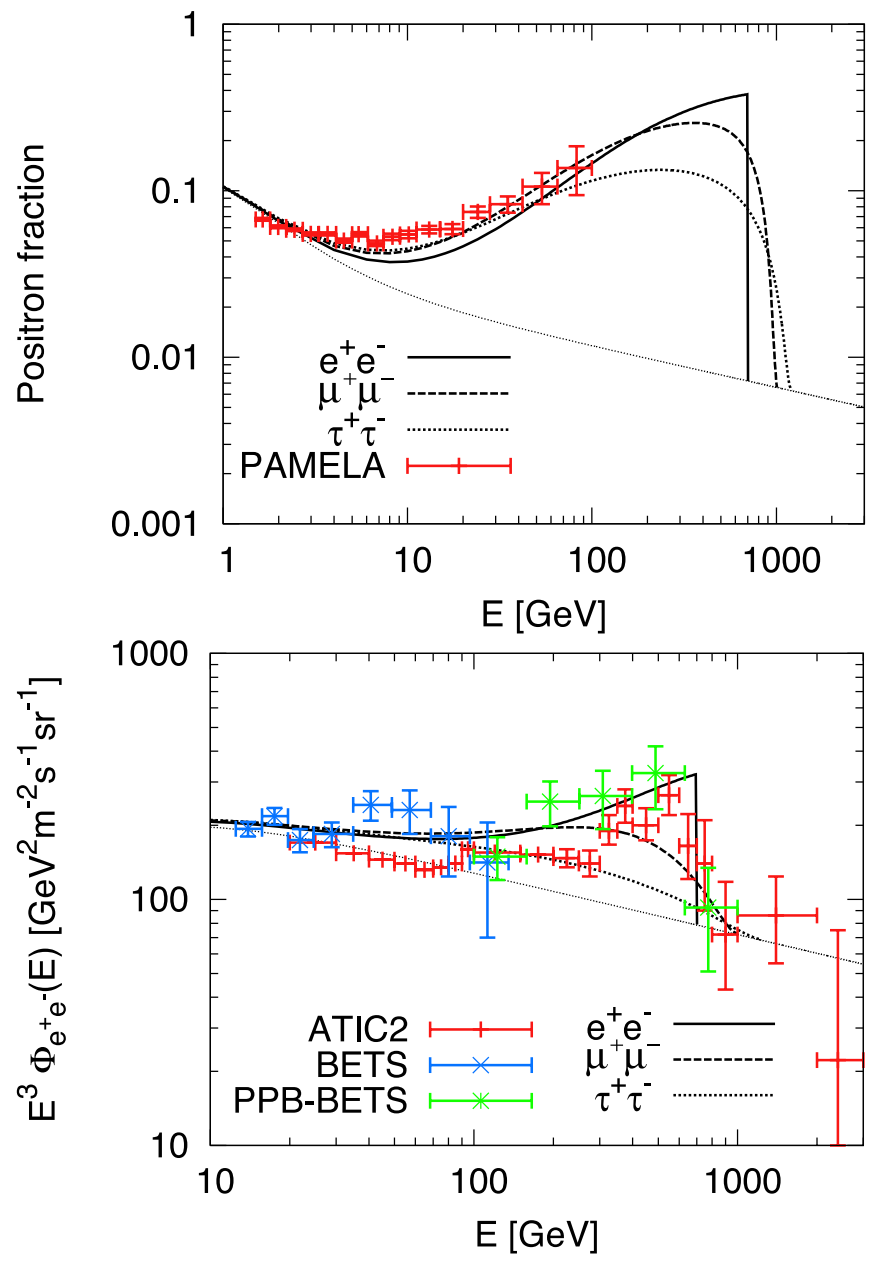

FIG. 1: The positron fraction (top) and total electron and positron flux from the annihilation (bottom) from dark matter annihilation. We take the mass and annihilation cross section as $m=0.7 \mathrm{TeV}$ and $\langle\sigma v\rangle=5 \times 10^{-24} \mathrm{~cm}^{3} \mathrm{~s}^{-1}$ for the mode into $e^{+} e^{-}$(solid), $m=1 \mathrm{TeV}$ and $\langle\sigma v\rangle=1.5 \times 10^{-23} \mathrm{~cm}^{3} \mathrm{~s}^{-1}$ for the mode into $\mu^{+} \mu^{-}$(dashed), $m=1.2 \mathrm{TeV}$ and $\langle\sigma v\rangle=$ $2 \times 10^{-23} \mathrm{~cm}^{3} \mathrm{~s}^{-1}$ for the mode into $\tau^{+} \tau^{-}$(dotted). Results of PAMELA, ATIC2, BETS and PPB-BETS are plotted.

for the case of decay. Here, $R_{\odot}=8.5 \mathrm{kpc}$ and $\rho_{\odot}=$ $0.3 \mathrm{GeVcm}^{-3}$ are the distance of the solar system from the Galactic center and local dark matter density near the solar system, $F$ collectively denotes the primary annihilation/decay mode (e.g., $\mu^{+} \mu^{-}$, etc.), and $d N_{F}^{\left(\nu_{i}\right)} / d E$ represents the neutrino spectrum arising from the final state $F$. The dependence on the dark matter halo density profile is contained in the remaining factor $\left\langle J_{n}\right\rangle_{\Omega}$, defined by

$$
\left\langle J_{n}\right\rangle_{\Omega}=\int \frac{d \Omega}{\Delta \Omega} \int_{\text {l.o.s. }} \frac{d l(\psi)}{R_{\odot}}\left(\frac{\rho(l)}{\rho_{\odot}}\right)^{n},
$$

where $l(\psi)$ is the distance from us along the direction $\psi$, which is the cone-half angle from the Galactic center within the range $0<\psi<\psi_{\max }$, and $\Delta \Omega$ (三 
$\left.2 \pi\left(1-\cos \psi_{\max }\right)\right)$ is the solid angle over which the neutrino flux is averaged. Typical values are $\left\langle J_{2}\right\rangle_{\Omega} \Delta \Omega \sim 10$ and $\left\langle J_{1}\right\rangle_{\Omega} \Delta \Omega \sim 0.4$ for $\psi_{\max }=5^{\circ}$, if the Navarro-FrenkWhite (NFW) halo density profile [17] is adopted. For the isothermal profile $\left\langle J_{2(1)}\right\rangle_{\Omega} \Delta \Omega$ is $\sim 2(0.4)$.

The best technique for detecting neutrinos from the Galactic center is observation of the up-going muons, which are produced in the rock below the detectors. The detectors in the northern hemisphere observe the upgoing muon induced by neutrinos from the Galactic center. The neutrino-induced muon flux is evaluated from the neutrino flux [9, 18] as

$$
\begin{aligned}
F_{\mu^{+} \mu^{-}}^{(\mathrm{ann})} & \simeq 5.9 \times 10^{-15} \mathrm{~cm}^{-2} \mathrm{~s}^{-1} \\
& \times \sum_{F} S_{F}\left(\frac{\langle\sigma v\rangle_{F}}{10^{-23} \mathrm{~cm}^{3} \mathrm{~s}^{-1}}\right)\left(\frac{\left\langle J_{2}\right\rangle_{\Omega} \Delta \Omega}{10}\right),
\end{aligned}
$$

for the case of annihilation and

$$
\begin{aligned}
F_{\mu^{+} \mu^{-}}^{(\mathrm{dec})} & \simeq 2.0 \times 10^{-15} \mathrm{~cm}^{-2} \mathrm{~s}^{-1}\left(\frac{m_{\chi}}{2 \mathrm{TeV}}\right) \\
& \times \sum_{F} S_{F}\left(\frac{\Gamma_{F}}{10^{-26} \mathrm{~s}^{-1}}\right)\left\langle J_{1}\right\rangle_{\Omega} \Delta \Omega,
\end{aligned}
$$

for the case of decay. Here we have defined $S_{F}$ through

$$
S_{F}=\sum_{\nu_{i}} \int_{E_{\min }}^{E_{\mathrm{in}}} \frac{d N_{F}^{\left(\nu_{i}\right)}}{d E} P_{\nu_{i} \nu_{\mu}}\left(\frac{E}{E_{\mathrm{in}}}\right)^{2} d E,
$$

where $E_{\text {in }}=m_{\chi}\left(m_{\chi} / 2\right)$ for the case of annihilation (decay), and $E_{\min }$ is the threshold energy above which the muons can be detected. $P_{\nu_{i} \nu_{\mu}}$ denotes the probability that the $\nu_{i}$ at the production is observed as $\nu_{\mu}$ at the Earth due to the effect of neutrino oscillation. The value of $S_{F}$ is summarized in a following table. These values are insensitive to the precise value of $E_{\min }$ as long as it is smaller than $100 \mathrm{GeV}$.

\begin{tabular}{|c||c|c|c|c|c|c|c|}
\hline & $\nu_{e} \bar{\nu}_{e}$ & $\nu_{\mu} \bar{\nu}_{\mu}$ & $\nu_{\tau} \bar{\nu}_{\tau}$ & $\mu_{R}^{-} \mu_{L}^{+}$ & $\mu_{L}^{-} \mu_{R}^{+}$ & $\tau_{R}^{-} \tau_{L}^{+}$ & $\tau_{L}^{-} \tau_{R}^{+}$ \\
\hline$S_{F}$ & 0.44 & 0.78 & 0.78 & 0.19 & 0.20 & 0.14 & 0.18 \\
\hline
\end{tabular}

Remarkably, the neutrino-induced muon flux is proportional to the second moment of the neutrino energy spectrum. It implies that the up-going muon detection is more sensitive to the energetic neutrinos. As the result, the muon flux is independent of the dark matter mass in the case of annihilation, and it is proportional to the mass in the case of decay. (See Eqs. (718).) This is a good news because ATIC/PPB-BETS indicate TeV scale dark matter mass, rather than of the order of $100 \mathrm{GeV}$, and such heavy dark matter models have benefits from the viewpoint of detection at the neutrino detectors.

In order to reproduce ATIC/PPB-BETS anomaly, dark matter annihilation/decay directly into leptons are more favored as mentioned above. Here, we assume for simplicity that the dark matter particle is $S U(2) \times U(1)$ singlet. In the case, monochromatic neutrinos are naturally generated when the dark matter annihilates/decays
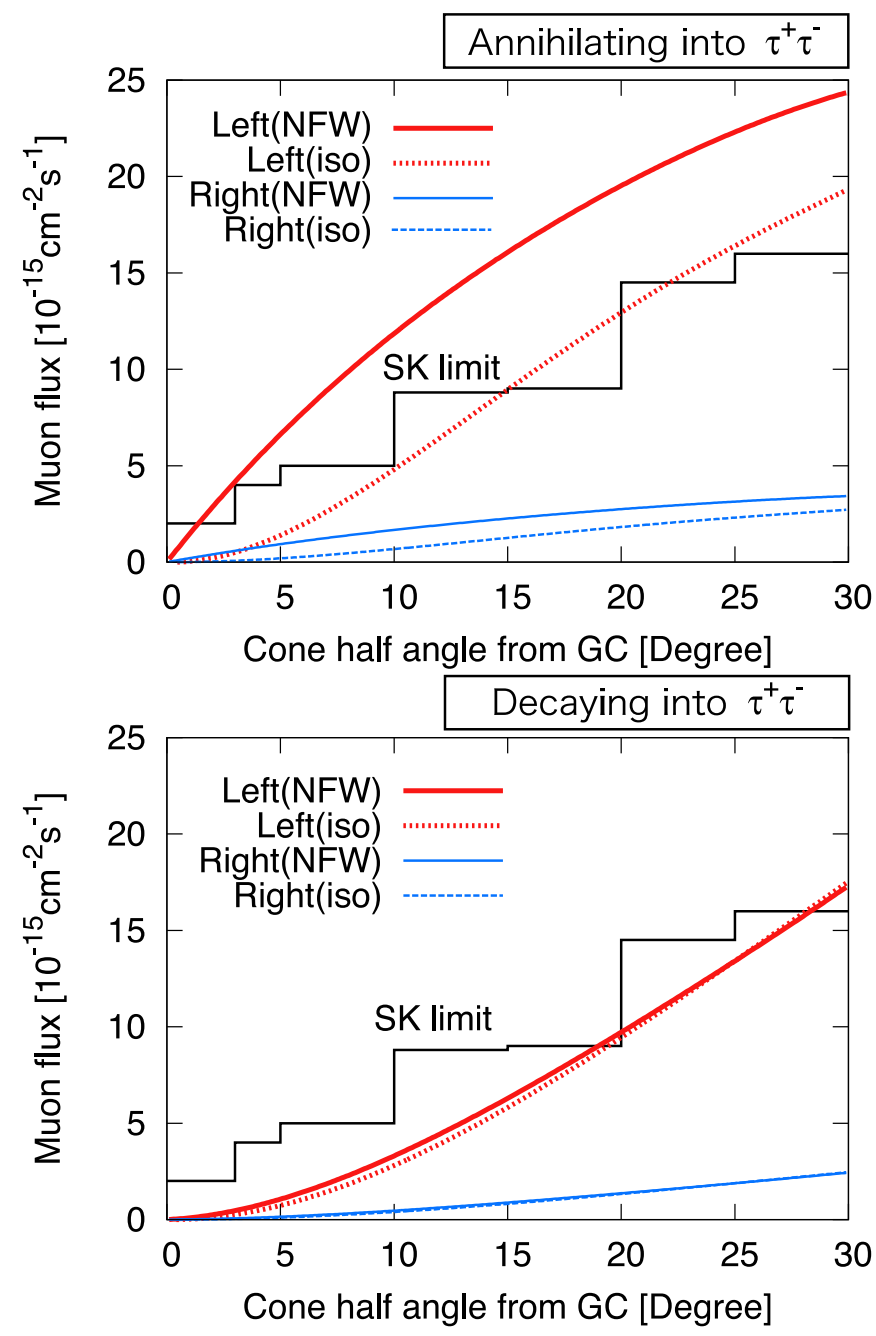

FIG. 2: Expected up-going muon flux from the dark matter annihilation (top) and decay (bottom) as a function of the cone half angle from the Galactic center. SK limits are also shown. Here, we assume the the dark matter annihilates/decays into left-handed leptons $\left(\tau_{L}^{-} \tau_{R}^{+}\right.$and $\left.\nu_{\tau} \bar{\nu}_{\tau}\right)$ shown by "Left" or right-handed leptons $\left(\tau_{R}^{-} \tau_{L}^{+}\right)$shown by "Right". Annihilating Dark matter models correspond to those used in Fig. 1. For the case of decaying dark matter, we used $\Gamma=1.5 \times 10^{-26} \mathrm{~s}^{-1}$ and $m=2.4 \mathrm{TeV}$. The dark matter density profile is assumed to be the NFW and isothermal profiles.

into left-handed charged leptons. On the other hand, the dark matter annihilating/decaying into only the righthanded charged leptons can produce neutrinos secondarily. Therefore, in the following, we consider two cases: dark matter annihilates (decays) into left-handed leptons (i.e., neutrinos and charged leptons) with same branching ratio, and into all right-handed-leptons (i.e., no direct production of neutrinos).

The muon flux from the dark matter annihilation (decay) is shown in the top (bottom) panel of Figs. 2[4] as a function of the cone half angle from the Galactic center, both for the isothermal and NFW profile and for the 

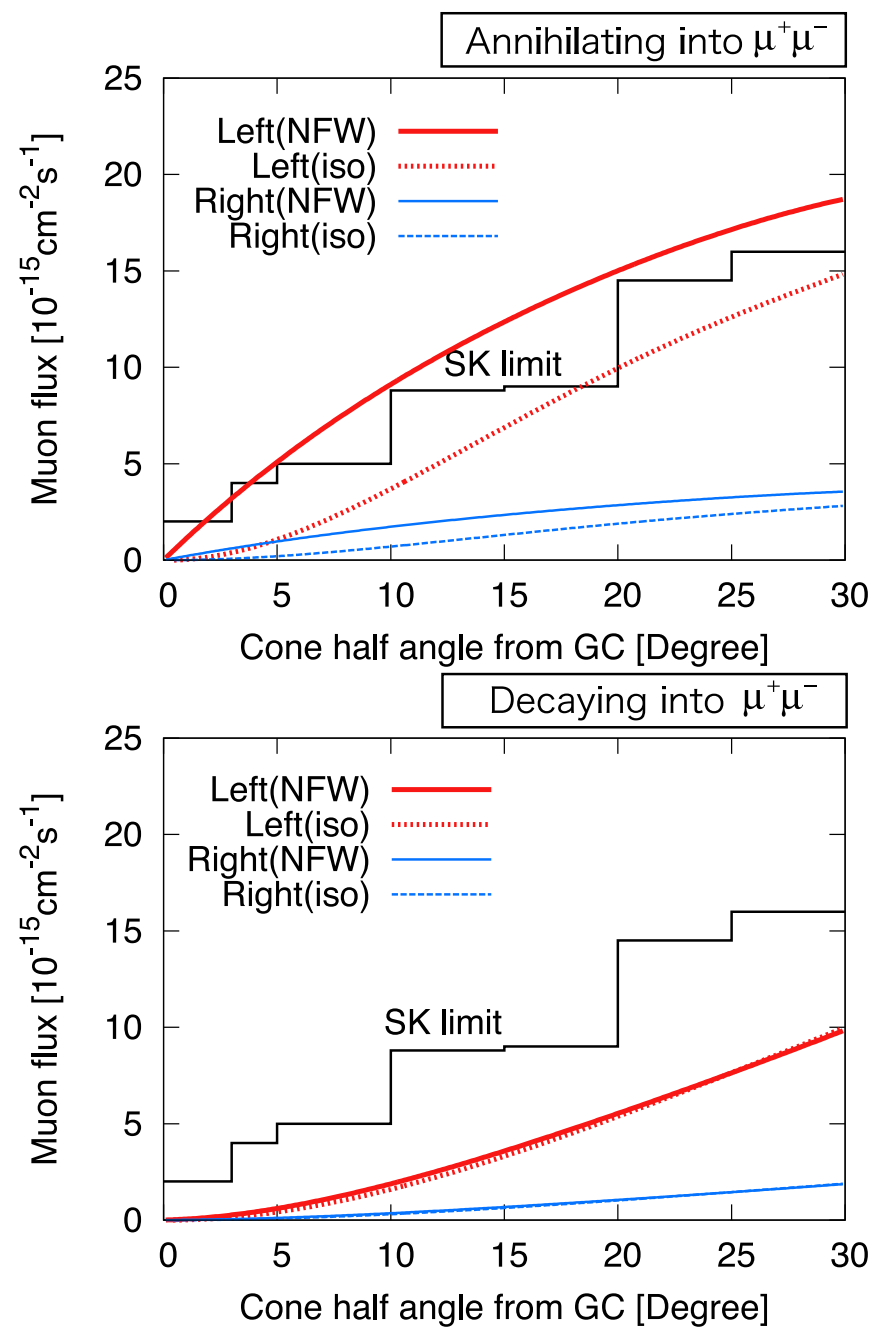

FIG. 3: Same as Fig. 2 but for annihilating (decaying) into $\mu^{-} \mu^{+}$. For the case of decaying dark matter, we used $\Gamma=$ $1 \times 10^{-26} \mathrm{~s}^{-1}$ and $m=2 \mathrm{TeV}$.

case of annihilation (decay) into $\tau^{+} \tau^{-}, \mu^{+} \mu^{-}$and $e^{+} e^{-}$. We have assumed same mass and cross section as those used in Fig. 1]so that produced charged leptons exhibit a good fit on the PAMELA and ATIC/PPB-BETS results. For the case of decaying dark matter, we used following parameters : $\Gamma=4 \times 10^{-27} \mathrm{~s}^{-1}$ and $m=1.4 \mathrm{TeV}$ for the case of $e^{+} e^{-}, \Gamma=1 \times 10^{-26} \mathrm{~s}^{-1}$ and $m=2 \mathrm{TeV}$ for the case of $\mu^{+} \mu^{-}, \Gamma=1.5 \times 10^{-26} \mathrm{~s}^{-1}$ and $m=2.4 \mathrm{TeV}$ for the case of $\tau^{+} \tau^{-}$. Resulting positron and electron flux looks quite similar to Fig. 1 In the case of annihilation (decay) into left-handed leptons, we have assumed the same annihilation (decay) rate into neutrino pairs. Also shown are limits from Super-Kamiokande (SK) 19]. It is found that if the neutrinos are directly produced by the annihilation/decay of the dark matter with the same rate as charged leptons, SK may already give constraints on some dark matter models such as those annihilating/decaying into $\tau^{+} \tau^{-}$and annihilating into $\mu^{+} \mu^{-}$. Notice that parameters chosen here (annihilation cross
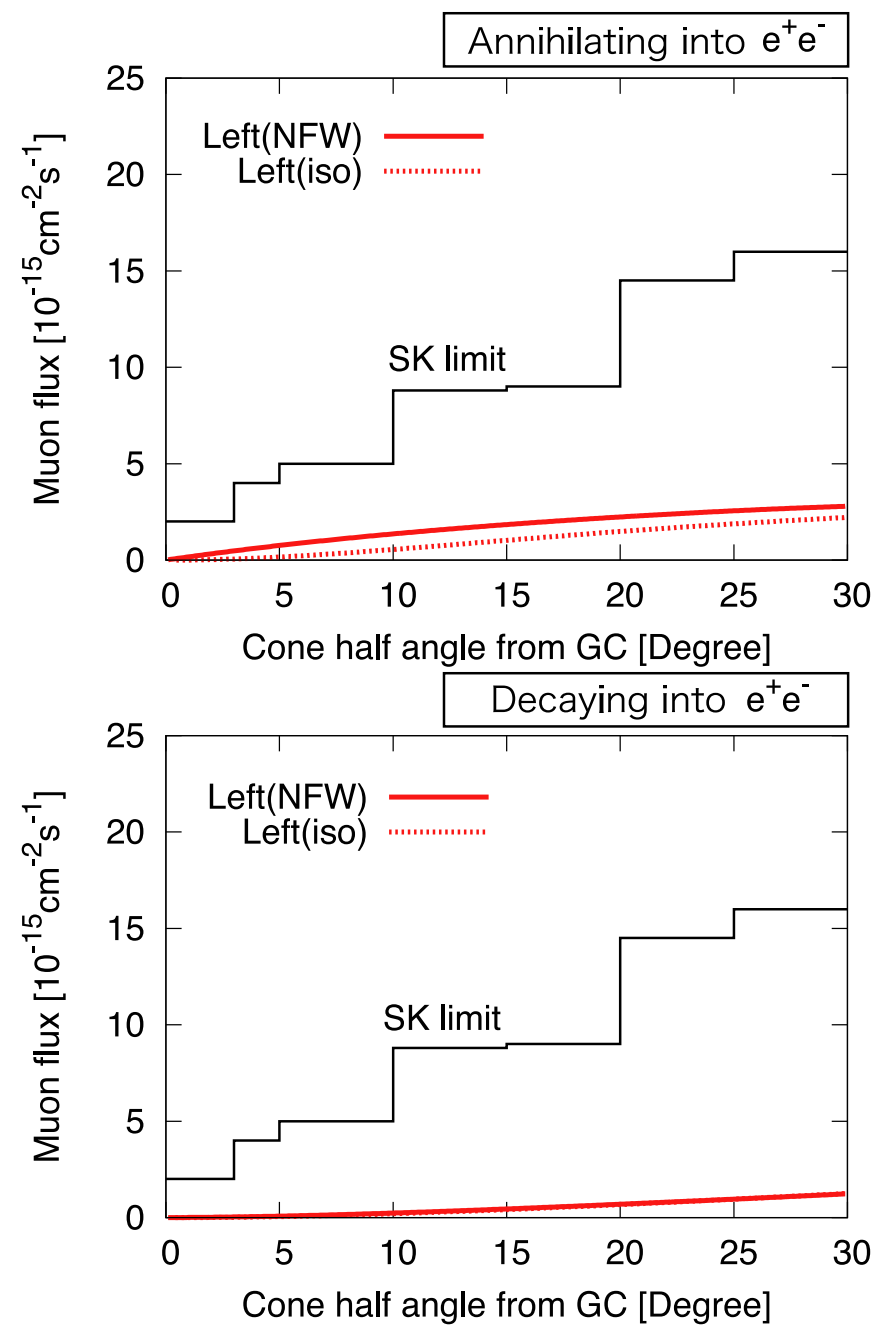

FIG. 4: Same as Fig. 2, but for annihilating (decaying) into $e^{-} e^{+}$. For the case of decaying dark matter, we used $\Gamma=$ $4 \times 10^{-26} \mathrm{~s}^{-1}$ and $m=1.4 \mathrm{TeV}$.

section, decay rate and mass) should be regarded as only representative values, and they include $\mathcal{O}(1)$ uncertainties in order to fit the PAMELA/ATIC results depending on dark matter models, which may either increase or decrease the resulting muon flux. Therefore, model-bymodel comparison should be performed in order to check a consistency with SK bound.

Some comments are in order. The final state leptons radiate gamma rays through the internal bremstrahlung or cascade decay processes, which should be compared with the gamma-ray flux observed by the HESS experiment [20]. In the case of annihilation, the constraint is severe and the PAMELA/ATIC results may be inconsistent with the HESS observation, if the cuspy density profile such as NFW profile is adopted [8]. This can be relaxed for the isothermal profile. On the other hand, the dependence of the neutrino flux on the density profile is rather weak since the SK looks at the Galactic center over wide angle. Thus it may be possible that neutrinos give 
more stringent constraint on the dark matter annihilation scenario. If only the PAMELA anomaly is taken into account, lighter dark matter is possible. In that case, the muon signals at neutrino detectors are suppressed since low-energy neutrinos have less potential to be converted into muons and less propagation length inside the Earth.

In the case of decaying dark matter, neutrino constraints are very useful since the gamma-ray flux gives only loose constraints even for the NFW profile [8]. In addition, neutrino-induced muon flux receives an extra enhancement factor proportional to the dark matter mass as shown in Eq. (8). Therefore, a decaying dark matter with a few $\mathrm{TeV}$, indicated by ATIC results, may have distinct signatures on the neutrino flux, rather than gamma rays.

It is noticed that a significant amount of neutrino flux is not expected in the pulsar scenario for the positron excess. Therefore, if we detect unknown neutrino signals from the Galactic center, the annihilation/decay scenario could be distinguished from the other astrophysical scenarios and be verified. The planned future mega-ton scale water tank detector, Hyper-Kamiokande, which is the extension of the SK, and kilo-meter size detector, such as KM3NeT, are expected to improve the current sensitivity of the SK by one or two orders of magnitude. It is recently discussed that the IceCube DeepCore, which is a planned extension of the IceCube, would also have sensitivity on high-energy neutrinos from the Galactic center 21]. These neutrino experiments will be very useful for confirming, distinguishing or excluding some of the dark matter models as an explanation of the recently observed cosmic electron/positron excesses.

\section{Acknowledgments}

The authors appreciate Prof. Kajita who encouraged them to evaluate the constraints on neutrino flux originated from the dark matter annihilation and decay. KN would like to thank the Japan Society for the Promotion of Science for financial support. This work is supported by Grant-in-Aid for Scientific research from the Ministry of Education, Science, Sports, and Culture (MEXT), Japan, No.14102004 (MK) and No. 20244037 and No. 2054252 (JH), and also by World Premier International Research Center InitiativeiWPI Initiative), MEXT, Japan. KK is supported in part by PPARC grant, PP/D000394/1, EU grant MRTN-CT2006-035863, the European Union through the Marie Curie Research and Training Network "UniverseNet".
[1] O. Adriani et al., arXiv:0810.4995 [astro-ph].

[2] J. Chang et al., Nature 456, 362 (2008).

[3] S. Torii et al., arXiv:0809.0760 [astro-ph].

[4] H. E. S. S. Collaboration, arXiv:0811.3894 [astro-ph].

[5] A. M. Atoian, F. A. Aharonian and H. J. Volk, Phys. Rev. D 52, 3265 (1995).

[6] D. Hooper, P. Blasi and P. D. Serpico, arXiv:0810.1527 [astro-ph]; P. D. Serpico, arXiv:0810.4846 [hep-ph]; H. Yuksel, M. D. Kistler and T. Stanev, arXiv:0810.2784 [astro-ph].

[7] L. Bergstrom, T. Bringmann and J. Edsjo, arXiv:0808.3725 [astro-ph]; M. Cirelli and A. Strumia, arXiv:0808.3867 [astro-ph]; V. Barger, W. Y. Keung, D. Marfatia and G. Shaughnessy, arXiv:0809.0162 [hep-ph]; C. R. Chen, F. Takahashi and T. T. Yanagida, arXiv:0809.0792 [hep-ph]; I. Cholis, L. Goodenough, D. Hooper, M. Simet and N. Weiner, arXiv:0809.1683 [hep-ph]; M. Cirelli, M. Kadastik, M. Raidal and A. Strumia, arXiv:0809.2409 [hep-ph]; N. ArkaniHamed, D. P. Finkbeiner, T. Slatyer and N. Weiner, arXiv:0810.0713 [hep-ph]; C. R. Chen and F. Takahashi, arXiv:0810.4110 [hep-ph]; F. Donato, D. Maurin, P. Brun, T. Delahaye and P. Salati, arXiv:0810.5292 [astro-ph]; I. Cholis, D. P. Finkbeiner, L. Goodenough and N. Weiner, arXiv:0810.5344 [astro-ph]; Y. Nomura and J. Thaler, arXiv:0810.5397 [hep-ph]; R. Harnik and G. D. Kribs, arXiv:0810.5557 [hep-ph]; P. f. Yin, Q. Yuan, J. Liu, J. Zhang, X. j. Bi and S. h. Zhu, arXiv:0811.0176 [hep-ph]; K. Ishiwata, S. Matsumoto and T. Moroi, arXiv:0811.0250 [hep-ph]; Y. Bai and Z. Han, arXiv:0811.0387 [hep-ph]; P. J. Fox and
E. Poppitz, arXiv:0811.0399 [hep-ph]; C. R. Chen, F. Takahashi and T. T. Yanagida, arXiv:0811.0477 [hepph]; arXiv:0811.3357 [astro-ph]; A. Ibarra and D. Tran, arXiv:0811.1555 [hep-ph]; J. Hall and D. Hooper, arXiv:0811.3362 [astro-ph]; I. Cholis, G. Dobler, D. P. Finkbeiner, L. Goodenough and N. Weiner, arXiv:0811.3641 [astro-ph].

[8] G. Bertone, M. Cirelli, A. Strumia and M. Taoso, arXiv:0811.3744 [astro-ph]; E. Nardi, F. Sannino and A. Strumia, arXiv:0811.4153 [hep-ph].

[9] As a review, see G. Jungman, M. Kamionkowski and K. Griest, Phys. Rept. 267, 195 (1996).

[10] K. Jedamzik, Phys. Rev. D 70, 083510 (2004) arXiv:astro-ph/0405583; J. Hisano, M. Kawasaki, K. Kohri and K. Nakayama, arXiv:0810.1892 [hep-ph].

[11] L. Covi, M. Grefe, A. Ibarra and D. Tran, arXiv:0809.5030 [hep-ph].

[12] E. A. Baltz and J. Edsjo, Phys. Rev. D 59, 023511 (1999) arXiv:astro-ph/9808243.

[13] J. Hisano, S. Matsumoto, O. Saito and M. Senami, Phys. Rev. D 73, 055004 (2006) arXiv:hep-ph/0511118.

[14] S. Torii et al., Astrophys. J. 559, 973 (2001).

[15] J. Hisano, S. Matsumoto and M. M. Nojiri, Phys. Rev. Lett. 92, 031303 (2004) arXiv:hep-ph/0307216; J. Hisano, S. Matsumoto, M. M. Nojiri and O. Saito, Phys. Rev. D 71, 063528 (2005) arXiv:hep-ph/0412403.

[16] J. F. Beacom, N. F. Bell and G. D. Mack, Phys. Rev. Lett. 99, 231301 (2007) arXiv:astro-ph/0608090; H. Yuksel, S. Horiuchi, J. F. Beacom and S. Ando, Phys. Rev. D 76, 123506 (2007) arXiv:0707.0196 [astro-ph]].

[17] J. F. Navarro, C. S. Frenk and S. D. M. White, Astro- 
phys. J. 462, 563 (1996) arXiv:astro-ph/9508025.

[18] S. Ritz and D. Seckel, Nucl. Phys. B 304, 877 (1988).

[19] S. Desai et al. [Super-Kamiokande Collaboration], Phys. Rev. D 70, 083523 (2004) [Erratum-ibid. D 70, 109901 (2004)] arXiv:hep-ex/0404025.

[20] F. Aharonian et al. [The HESS Collaboration], Astron.
Astrophys. 425, L13 (2004) arXiv:astro-ph/0408145. F. Aharonian et al. [H.E.S.S. Collaboration], Nature 439, 695 (2006) arXiv:astro-ph/0603021.

[21] D. F. Cowen [IceCube Collaboration], J. Phys. Conf. Ser. 110, 062005 (2008). 